\title{
Nuevas tecnologías de recobro químico y su aplicabilidad en yacimientos colombianos
}

\author{
C. Delgadillo ${ }^{1 *}$; C. Espinosa ${ }^{2} ;$ V. Díaz ${ }^{2}$; M. Ruiz : E. Manrique $^{1}$
}

doi: http://dx.doi.org/10.18273/revfue.v18n2-2020001 @) (1)

Forma de citar: Delgadillo, C., Espinosa, C., Díaz, V., Ruiz, M., \& Manrique, E. (2020). Nuevas tecnologías de recobro químico y su aplicabilidad en yacimientos colombianos. Revista Fuentes, el reventón energético, 18(2), 9-24. https://doi.org/10.18273/revfue.v18n2-2020001

\section{Resumen}

En la última década se ha avanzado en la implementación de procesos de recobro químico en Colombia, incluyendo pilotos de inyección de geles obturantes para conformance químico, geles de dispersión coloidal, polímeros, álcali y surfactantes. Estos procesos han sido de marcada relevancia tanto para la promesa de incrementar el factor de recobro de los campos colombianos, como para revisar campos en sus planes de abandono mediante la aplicación de nuevas tecnologías. No obstante, estudios experimentales a condiciones de yacimiento han evidenciado que existen limitaciones para la aplicación de estas tecnologías principalmente en lo referente a: calidad del agua de inyección, salinidad del agua de formación, temperatura, entre otras; esto dificulta establecer la viabilidad técnica y/o económica de su implementación en activos con alto potencial de reservas y posibles escenarios de expansión.

Por lo anterior y debido a la necesidad de incrementar las reservas de crudo, contribuyendo al entendimiento y desarrollo del recobro mejorado de hidrocarburos en el país, este artículo presenta una revisión de las limitaciones de las tecnologías de recobro químico convencionales, las oportunidades de las nuevas tendencias que permiten superar limitaciones técnicas y operacionales; además, de una estimación del potencial de las tecnologías emergentes evaluadas en el contexto nacional. Para esto, mediante la comparación de las ventanas operacionales de las tecnologías con las propiedades petrofísicas de los campos colombianos, fue posible establecer el potencial de incremento de reservas por efecto de la aplicación de nuevas tecnologías.

Adicionalmente, se identificaron las necesidades en el diseño e instalación de nuevas facilidades para el tratamiento, almacenamiento e inyección de los químicos y sus costos asociados; teniendo como premisa que la aplicación de una nueva tecnología de recobro en este caso está condicionada a una viabilidad técnicoeconómica que permita aprovechar las facilidades existentes en campo optimizando en los posibles CAPEX (del inglés Capital Expenditures) asociados a los proyectos.

Palabras clave: recobro mejorado, procesos químicos, nuevas tecnologías, parámetros de screening, ventanas de operación.

\section{New chemical recovery technologies and their applicability in Colombian fields}

\begin{abstract}
In the last decade, progress has been made in the implementation of chemical recovery processes in Colombia, including sealing gels injection pilots for chemical conformance, colloidal dispersion gels, polymers, alkali and surfactants. These processes have been of marked relevance both for the promise of increasing the Colombian fields recovery factor and for reviewing fields in their abandonment plans through the application of new technologies. However, experimental studies at reservoir conditions have shown that there are limitations for the application of these technologies, mainly in relation to: injection water quality, formation water salinity,
\end{abstract}

\footnotetext{
${ }^{1}$ Ecopetrol, Instituto Colombiano del Petróleo. (*) claudia.delgadillo@ecopetrol.com.co

${ }^{2}$ Universidad Industrial de Santander
} 
temperature, among others; This makes it difficult to establish the technical and / or economic viability of its implementation in assets with high reserve potential and possible expansion scenarios.

For to the above, and due the need to increase crude oil reserves, contributing to the understanding and development of enhanced oil recovery in the country; this paper presents a review of the limitations of conventional chemical recovery technologies, the opportunities of emerging technologies that allow overcoming technical and operational limitations and an estimate of the emerging technologies potential evaluated in the national context. For this, by comparing the operational windows of the technologies with the petrophysical properties of the Colombian fields, it was possible to establish the potential for increased reserves due to the application of new technologies. In addition, the needs in the design and installation of new equipment for the storage, chemicals injection and their associated costs were identified, taking as a premise that the application of a new recovery technology, in this case, is conditional on technical - economic feasibility, that allow to take advantage of the existing facilities in the field optimizing the possible CAPEX (Capital Expenditures) associated with the projects.

Keywords: enhanced oil recovery, chemical processes, emerging technologies, screening parameters, operation windows.

\section{Introducción}

Colombia es un país con pocas aplicaciones de técnicas de recobro mejorado si se compara con países como Canadá, China, Estados Unidos o Argentina donde la mayoría de sus yacimientos se encuentran en procesos maduros de inyección de agua y, por tanto, son líderes en explotación usando técnicas de recobro mejorado (Pye, 1964; Sandiford, 1964; Yang et al., 2004; Sheng, 2010; Alvarado y Manrique 2010). Sin embargo, las condiciones geológicas en estos países, y las características de los hidrocarburos allí extraídos, son diferentes a las encontradas en Colombia, lo que ha dificultado la transferencia tecnológica de los métodos de recobro mejorado en el país (Barrero et al, 2007; Amaya et al, 2010).

Por otra parte, de acuerdo con Alvarado y Manrique (2010) en los últimos años ha incrementado el interés por la exploración y desarrollo de yacimientos no convencionales, por lo que la importancia de procesos EOR (del inglés Enhanced Oil Recovery) ha cambiado, haciendo que su tendencia sea focalizarse en regiones específicas del mundo. Esto no significa para el país o la compañía un retroceso, por el contrario, implica la obligatoriedad de asumir el liderazgo en el área, desarrollar sus propios proyectos, investigar de primera mano y resolver en el corto/mediano plazo los retos que el recobro mejorado ha dejado sobre la mesa (Maya et al, 2010; Castro et al, 2014).

A la luz de este reto y la eventual disminución de la ventana de oportunidad del recobro mejorado ya sea por variaciones de precio de crudo o por la aparición de nuevas fuentes energéticas, se debe reaccionar más rápido para alcanzar soluciones efectivas y oportunas. En este sentido este trabajo busca, partiendo de las experiencias adquiridas en los recientes 10 años validando procesos CEOR (del inglés Chemical EOR) tradicionales, identificar sus limitaciones o restricciones en las condiciones de los yacimientos colombianos, y conocer aquellas nuevas tecnologías con mayor aplicabilidad. Con base en estas experiencias y estudios previos (DelgadilloAya et al, 2018), las condiciones de aplicación de las nuevas tecnologías de recobro y las propiedades de los yacimientos colombianos se construye el trabajo presentado a continuación.

\section{Tecnologías Convencionales Vs Tecnologías Emergentes}

En los campos petroleros colombianos, durante laúltima década se han diseñado, implementado y monitoreado pilotos, además de evaluar alternativas de expansión, de diferentes tecnologías CEOR. Se han ejecutado pilotos de inyección de soluciones poliméricas en los campos Yariguí - Cantagallo, Casabe, Palogrande - Cebú y Chichimene, cada uno con características específicas que hacen que el proceso sea característico en cada campo en términos tanto operativos como de las condiciones de los químicos usados (Castro et al, 2013; Maya et al, 2015; Castro et al, 2016; CastroGarcia et al, 2016; Perez et al, 2017).

Adicionalmente, se han empleado tecnologías para bloqueo de zonas ladronas y control de movilidad a grandes distancias del pozo (conformance profundo) como estrategias de optimización de proyectos de inyección de agua en los campos Tello y Dina Cretáceo, respectivamente. Ambos proyectos estuvieron orientados a mejorar la eficiencia volumétrica de barrido en tres patrones del campo Tello y en cuatro patrones del campo Dina Cretáceo. Por otra parte, se realizó 
un piloto de inyección de álcali-surfactante-polímero (ASP) en el campo San Francisco, su implementación buscaba desplazar el banco de aceite remanente no móvil a la inyección de agua convencional, mediante la reducción de la saturación de aceite residual y el control de movilidad. Estos proyectos han sido bien documentados en la literatura. (Maya-Toro et al, 2012; Maya et al, 2014; Vanegas et al, 2019).

Teniendo en cuenta la evaluación y análisis de resultados de los métodos mencionados antes, fue posible identificar una estrecha ventana de aplicación para los métodos de recobro convencionales en los campos colombianos. Por tanto, es necesario realizar un análisis de las restricciones de estos métodos, junto con las posibles nuevas tecnologías que las pueden contrarrestar y su aplicación en los campos colombianos. A continuación, se presenta este análisis para el caso de tecnologías de polímeros y surfactantes, siendo estas tecnologías, de recobro químico, las más aplicadas en el mundo.

Inyección de polímeros. La inyección de polímero es la técnica de recobro mejorado que ha sido la más implementada dentro de la subdivisión CEOR en los últimos años (Rosenkilde, Brakstad y Smith, 2016). Consiste en aumentar la viscosidad de la solución acuosa inyectada mediante la adición de moléculas de polímero para así lograr una mejor relación de movilidad entre el petróleo y el agua (Sorbie, 1991; Sheng, 2010). Con esto, se busca mitigar los efectos de la canalización heterogénea de agua y la digitación viscosa, mejorando la eficiencia del barrido volumétrico $\mathrm{y}$, en consecuencia, la recuperación de petróleo remanente del proceso de inyección de agua (Lake, 1996).

Los polímeros utilizados en procesos de recobro mejorado son, mayormente, las poliacrilamidas parcialmente hidrolizadas (HPAM) y sus derivados (Seright, 2016; Albonico \& Lockhart, 1993; LizcanoNino et al 2020). La HPAM posee propiedades importantes (hidrofílica y soluble en agua, incrementa la viscosidad del agua, entre otras) que la hacen un buen candidato para procesos EOR; sin embargo, presenta limitaciones en su rendimiento en condiciones de alta temperatura y salinidad, donde las moléculas del polímero pueden configurarse de forma aleatoria y generar una disminución significativa de la viscosidad (Wu et al, 2012; Albonico \& Lockhart, 1993; Molano, et al 2014; Pinto, M. S. et al 2018; Sun G, et al 2018).

Adicionalmente, las principales restricciones de la HPAM están relacionadas con la degradación, ya sea mecánica, química, térmica o biológica, que sufre desde su preparación, el transporte hacia el yacimiento y su flujo a través del medio poroso; en este último, existen además dos fenómenos que afectan fuertemente el desempeño de la inyección de soluciones poliméricas: la retención del polímero en la superficie de la roca (entrampamiento mecánico) y la adsorción química. Estos fenómenos dependen de la química del polímero, la composición de la roca, la temperatura, la salinidad y el pH del agua de formación (Kamal et al, 2015; Hernandez, et al 2018). En la tabla 1, se resumen las limitaciones de la inyección de polímeros HPAM y la condición que las genera.

Tabla 1. Limitaciones de los polímeros HPAM.

\begin{tabular}{cc}
\hline Limitaciones & Causa \\
\hline Degradación mecánica & Esfuerzos de corte \\
\hline Degradación química & Interacción con $\mathrm{O}_{2}$ \\
Contenido de hierro & \begin{tabular}{c} 
(precipitación)* \\
\hline Degradación térmica
\end{tabular} \\
\hline Degradación biológica & Temperatura máxima $80^{\circ} \mathrm{C}^{* *}$ \\
\hline Entrampamiento mecánico & Proliferación de bacterias sulfato reductoras \\
\hline Adsorción química & Tamaño de la partícula (radios hidrodinámicos) \\
\hline
\end{tabular}

*(Zaitoun y Potie, 1983)

**(Kamal et al, 2015) 
A partir de las limitaciones técnicas mencionadas, en los últimos años las investigaciones se han centrado en el desarrollo de nuevos polímeros con buen rendimiento en condiciones de alta temperatura y alta salinidad (HTHS) (Albonico \& Lockhart, 1993; Thomas, 2008; Wu et al, 2014; Wu et al, 2015, DelgadilloAya et al, 2018; Araujo \& Araujo, 2018). Las nuevas tecnologías de inyección de polímeros proponen un incremento en su resistencia mediante la adición de nuevos monómeros, funcionalizaciones en HPAM (El-Hoshoudy et al, 2017) o polímeros mejorados con nano partículas (Bennetzen \& Mogensen, 2014; ShamsiJazeyi et al, 2014), thermo thickening polymers (Shaefer et al, 2011) y biopolímeros (Quadri et al, 2015; Sveistrup et al, 2016), entre otras modificaciones posibles. A continuación, se resumen las características de estos polímeros mejorados:

- Modificaciones de HPAM. Permiten suplir las limitaciones relacionadas con el alto peso molecular, sensibilidad al cizallamiento, presencia de sales, iones divalentes y alta temperatura (Kamal et al, 2015). La inclusión de monómeros, nano partículas o funcionalidades hidrofóbicas ofrece un conjunto de posibilidades para mejorar variables que influyen en el factor de recobro obtenido de los yacimientos; ya sea la disminución en la tensión interfacial agua-aceite, mejora en la viscosidad obtenida con polímeros, aumento en la eficiencia de desplazamiento en core flooding, entre otras (Wu et al, 2016).

- Polímeros asociativos. Son copolímeros aniónicos solubles en agua y modificados con grupos hidrófobos. Muestran un comportamiento superior en comparación con la HPAM al generar mayor viscosidad con menos concentración, especialmente a temperatura elevada $y$ en presencia de iones divalentes $(\mathrm{Ca}++$ and $\mathrm{Mg}++)$ (Reichenbach-Klinke, Stavland, \& Strand, 2016; Taylor y Nasr-El-Din, 1998; Dupuis et al, 2010).

- Biopolímeros. Algunos de los productos comerciales de origen biológico como la goma Xanthan, el Schizophyllan, la Hidroxietilcelulosa y el Escleroglucano, han resurgido como posibles candidatos por las excelentes propiedades que exhiben al someterlos a alta salinidad, alta temperatura, altos esfuerzos de corte y a la presencia de diversos iones. De acuerdo con Jensen (2018), la goma Xanthan presenta limitaciones a temperaturas superiores a $\operatorname{los} 75^{\circ} \mathrm{C}$, y puede mostrar pérdida de inyectividad debido a taponamiento del medio poroso provocado por las impurezas propias del proceso de producción del bioproducto (Jensen, Kadhum y Kozlowicz, 2018). Por su parte el Schizophyllan, es un polisacárido que ha mostrado eficacia como viscosificante $\mathrm{y}$ estabilidad a temperaturas y salinidades altas (Leonhardt et al., 2014). Por último, el Escleroglucano es un biopolímero que debido a su conformación con estructura molecular de triple hélice y a su naturaleza no iónica logra una viscosidad estable a altas durezas, salinidades y temperaturas de hasta $130^{\circ} \mathrm{C}$ (Sveistrup et al, 2016; Viñarta et al, 2013).

Inyección de surfactantes. Los surfactantes son moléculas anfifílicas, que tienen una parte hidrofóbica no polar, constituida principalmente por una cadena hidrocarbonada o fluorocarbonada, la cual está unida a una porción iónica o polar (hidrofílica). La parte hidrofílica puede ser no-iónica, iónica o anfotérica (Tadros, 2007). A baja concentración tienen la propiedad de adsorberse en la superficie o interfaz del sistema, disminuyendo considerablemente la energía interfacial, aumentando el número capilar y logrando movilizar el petróleo atrapado en el medio poroso por fuerzas capilares, permitiendo finalmente, su desplazamiento por el fluido inyectado (Spildo, Johannessen y Skauge, 2012).

Un surfactante candidato para aplicar en procesos EOR debe reunir las siguientes características: buena estabilidad térmica, habilidad para disminuir la tensión interfacial a valores ultra bajos, baja retención en la roca, tolerancia a la salinidad, compatibilidad con otros químicos inyectados en la formación y disponibilidad comercial a un costo que viabilice económicamente los proyectos (Kamal, Hussein y Sultan, 2017). Los factores que afectan el comportamiento de fase, la tensión interfacial y la alteración de la mojabilidad están relacionados principalmente con la retención del surfactante en la superficie de la roca, que se maximiza cuando se alcanza la concentración micelar crítica, por encima de la cual, la adsorción no aumenta significativamente. La retención puede ser causada por precipitación, adsorción (principal causa de pérdida de surfactante en el medio poroso) o entrampamiento de fase, y afecta enormemente la economía de un proyecto EOR con surfactantes.

La adsorción del surfactante ocurre principalmente por las interacciones electrostáticas y de atracción repulsión (Van Der Waals) entre el químico y algunos minerales de la roca. Igualmente, se ve afectada por 
el $\mathrm{pH}$ del agua de formación, el número ácido del crudo y la temperatura del yacimiento. Por otra parte, el comportamiento de fase se ve afectado por la temperatura del yacimiento y la salinidad del agua de formación (Kamal et al, 2017). En la tabla 2, se resumen las limitaciones de la inyección de surfactantes y la condición que las genera.

Tabla 2. Limitaciones de los surfactantes convencionales

\begin{tabular}{|c|c|}
\hline Limitaciones & Causa \\
\hline \multirow{3}{*}{ Alta adsorción química } & Interacciones electrostáticas y de Van Der Waals \\
\hline & pH de la formación, \\
\hline & Salinidad \\
\hline Superficie de separación ente fases & Alta temperatura \\
\hline Presencia de precipitados & Presencia de iones divalente \\
\hline
\end{tabular}

Combinaciones surfactante-polímero (SP) y álcali-surfactante-polímero (ASP). La inyección de surfactantes se combina con la inyección de un agente de alta viscosidad, para lograr barrer el crudo liberado por la disminución de la tensión interfacial; generalmente, se usan polímeros (Sheng, Leonhardt y Azri, 2015). Se combinan entonces dos mecanismos: el mecanismo de recuperación por la inyección de polímero, basado en la disminución de la diferencia de movilidad entre el fluido desplazante y el fluido desplazado, y el mecanismo de recuperación con la inyección de surfactante, basado en la disminución de la tensión interfacial.

Una de las variaciones de esta técnica es la inyección ASP, en donde el álcali (generalmente un hidróxido como $\mathrm{NaOH}$ o carbonato como $\mathrm{Na}_{2} \mathrm{CO}_{3}$ ) reacciona con los componentes ácidos naturalmente presente en el crudo, formando tensoactivos (surfactantes naturales) in situ (Olajire, 2014; Thomas, 2008). Además, es usado como inhibidor de adsorción de los surfactantes aniónicos sobre la roca (areniscas principalmente), actuando como un agente de sacrificio ya que su costo es mucho menor comparado con el del surfactante. Sin embargo, esto genera otros problemas como la precipitación de carbonatos en el yacimiento por incompatibilidad con el agua de la formación, lo cual debe evaluarse en cada caso particular (ShamsiJazeyi, Verduzco y Hirasaki, 2014).

La combinación SP y/o ASP considera aspectos como las interacciones entre polímero y surfactante, el fenómeno de entrampamiento de fase, la adsorción sobre las rocas y la separación cromatográfica de los diversos componentes por la combinación de dos o más surfactantes (Sheng, 2010). La suma de estas condiciones y las restricciones propias de la inyección de surfactantes, ha motivado el surgimiento de tecnologías denominadas smart surfactant (Song et al, 2016), surfactantes visco-elásticos (Raffa, Broekhuis y Picchioni, 2016) o surfactantes poliméricos (Raffa et al, 2015). En este sentido, los surfactantes poliméricos son macromoléculas que contienen partes hidrófilas e hidrófobas en su estructura. A diferencia de los surfactantes tradicionales la naturaleza macromolecular de estos sistemas permite una variedad mucho mayor de estructuras; se puede derivar de la polimerización de un monómero tensoactivo (polioles) o mediante la copolimerización de un monómero hidrófobo y un monómero hidrófilo (Raffa et al, 2015).

\section{Características de los yacimientos colombianos}

Con el objetivo de realizar una valoración comparativa de los métodos descritos antes y su posible aplicación en los campos colombianos, a continuación, se presenta un estado general de las características de 275 campos colombianos, contenidos en una base de datos propia de Ecopetrol. En el país se cuenta con 23 cuencas sedimentarias y 16 estructuras principales distribuidas en todo el país. Actualmente, 11 cuencas contribuyen a la producción de hidrocarburos, de las cuales seis se consideran maduras (Llanos orientales, Valle Superior del Magdalena, Valle Medio del Magdalena, Putumayo-Caguán, Catatumbo, Cordillera oriental), y las otras cinco son consideradas cuencas emergentes (Guajira, Guajira Offshore, Cesar-Ranchería, SinúSan Jacinto, Valle Inferior del Magdalena). Por otra parte, se desarrollan trabajos de exploración en siete cuencas adicionales que son consideradas Frontera 
(Los Cayos, Urabá, Chocó, Chocó Offshore, Tumaco, Tumaco Offshore, Cauca-Patía). (Barrero et al, 2007; ANH, 2010).

El principal yacimiento presente en los campos colombianos es la formación Carbonera, la cual se encuentra en la cuenca de los Llanos Orientales y representa el $25 \%$ de los yacimientos en el país. La siguiente formación con mayor presencia es Caballos, que se encuentra en la cuenca Caguán-Putumayo y representa el $12.9 \%$ de los yacimientos del país. En tercer lugar, se encuentra la formación Mugrosa, presente en los campos de la cuenca del Valle Medio del Magdalena y que representa un $12.5 \%$ del total de los yacimientos.

En la figura 1 se observa la distribución de los yacimientos en Colombia de acuerdo con la cantidad de crudo en sitio, en la cual se puede observar que el país no cuenta con un gran número de yacimientos gigantes; sin embargo, en ellos se concentra más del $50 \%$ de crudo en sitio disponible en el país. Si se incluyen los campos grandes, que contienen $29.5 \%$ del crudo en sitio, se tiene que los yacimientos grandes y gigantes concentran más del $80 \%$ de crudo en sitio del país.

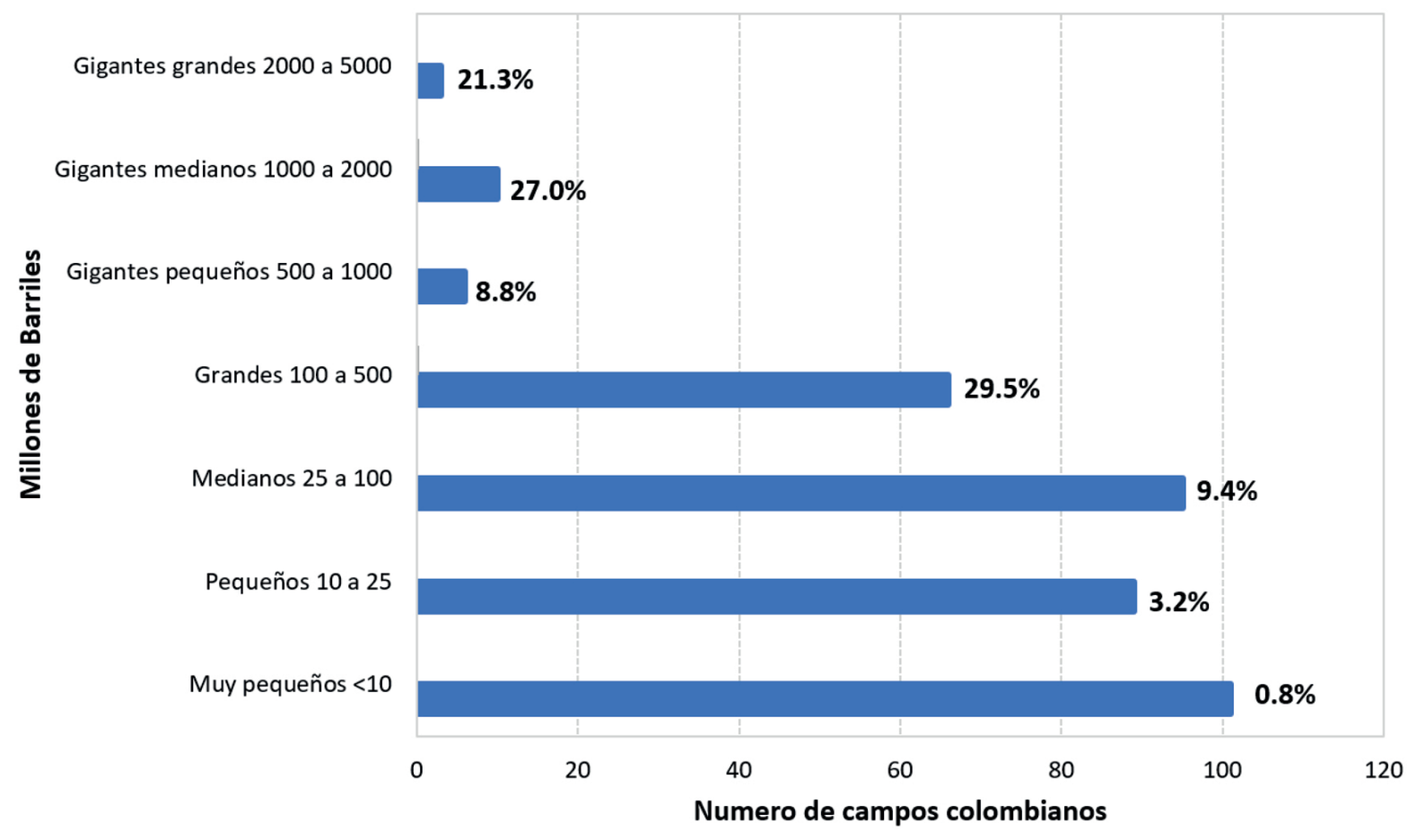

Figura 1. Distribución de los yacimientos colombianos según su cantidad de crudo en sitio

El petróleo original en sitio (OOIP, del inglés) en Colombia asciende a valores cercanos a los 48.000 millones de barriles, los cuales se encuentran distribuidos en los yacimientos que producen de las formaciones: Carbonera (20.3\%), Guadalupe (13.7\%) y Mugrosa (10.2\%) (British Petroleum, 2017; Ecopetrol S.A, 2010). No obstante, existen algunos yacimientos que contienen una cantidad de crudo en sitio considerable (entre 200 y 700 millones de barriles) y que por sus características resultan atractivos para la aplicación de procesos EOR. Estos yacimientos se encuentran en los campos Palagua, Orito, Tibú, Apiay y Caipal. En la figura 2 se observa la distribución del crudo en sitio de las formaciones más grandes del país (más del 1\% del OOIP); ésta figura permite identificar los yacimientos que contienen los mayores volúmenes de hidrocarburos en sitio y en donde podría encontrarse el mayor potencial para incrementar reservas mediante la aplicación de procesos de recobro mejorado (Ecopetrol S.A., 2010). 


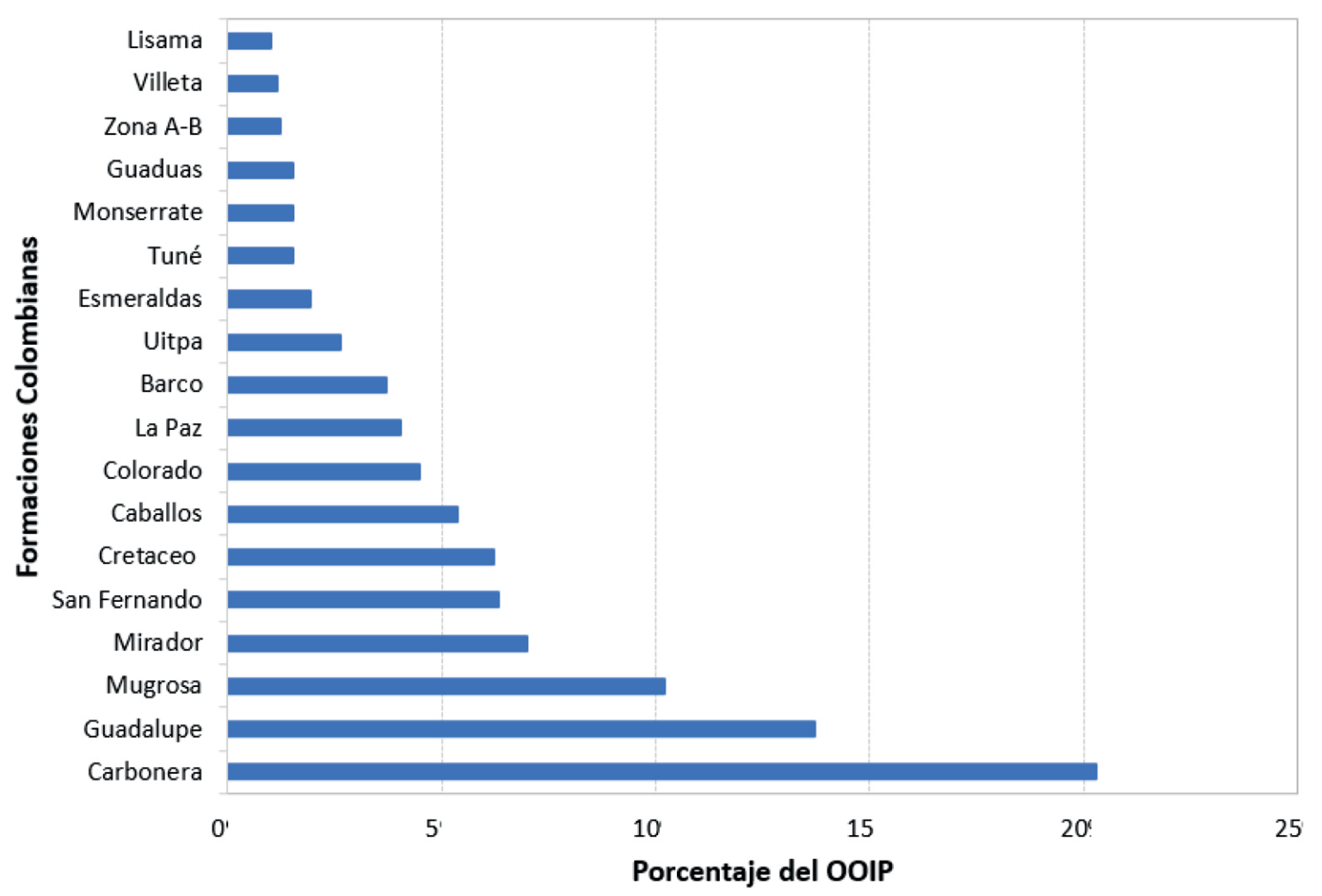

Figura 2. Distribución del crudo en sitio de las formaciones colombianas

Tabla 3. Propiedades de las principales formaciones geológicas del país

\begin{tabular}{|c|c|c|c|c|c|c|c|}
\hline $\begin{array}{l}\text { Ranking } \\
\text { OOIP }\end{array}$ & Formación & $\begin{array}{c}\text { Temperatura } \\
\left({ }^{\circ} \mathbf{F}\right)\end{array}$ & $\begin{array}{c}\text { Porosidad } \\
(\%)\end{array}$ & $\begin{array}{c}\text { Permeabilidad } \\
\text { (md) }\end{array}$ & API & $\begin{array}{l}\text { Viscosidad } \\
\text { Crudo (cp) }\end{array}$ & $\begin{array}{l}\text { Saturación } \\
\text { crudo }(\%)\end{array}$ \\
\hline \multirow{2}{*}{1} & \multirow{2}{*}{ Carbonera } & $94-290$ & $4.0-31.0$ & $1-30000$ & $12.7-44$ & $0.6-1200$ & $25-80$ \\
\hline & & $(192)$ & $(25.5)$ & (2233) & (29) & (32) & (57.9) \\
\hline \multirow{2}{*}{2} & \multirow{2}{*}{ Guadalupe } & $105-280$ & $2.0-30.0$ & $1-4000$ & $12.5-40.0$ & $0.2-150$ & $51.7-70$ \\
\hline & & (195) & $(16.2)$ & $(617)$ & (29.6) & $(23.4)$ & (63) \\
\hline \multirow{2}{*}{3} & \multirow{2}{*}{ Mugrosa } & $65-174$ & $8.3-30.0$ & $1-1000$ & $12.5-42.0$ & $0.5-10000$ & $36-70$ \\
\hline & & $(126)$ & $(18.1)$ & (198) & $(25.3)$ & $(478)$ & (56.4) \\
\hline 4 & $\begin{array}{c}\text { San } \\
\text { Fernando } \\
\end{array}$ & $180-250$ & $5.0-22.0$ & $50-4000$ & $7.0-14.5$ & $8.5-1000$ & $68.5-80$ \\
\hline 5 & Cretáceo & $212-230$ & $18-27$ & $200-2000$ & 29.4-33.6 & $1.8-4.9$ & $23-52$ \\
\hline \multirow{2}{*}{6} & \multirow{2}{*}{ Mirador } & $150-275$ & $2.0-36.0$ & $5-6000$ & $16-50$ & $0.02-30$ & $35.1-86$ \\
\hline & & $(222)$ & (15.3) & (1509) & $(30.7)$ & $(4.5)$ & (55.3) \\
\hline \multirow{2}{*}{7} & \multirow{2}{*}{ Caballos } & $111-265$ & $1.0-20.0$ & $1-3000$ & $16.0-38.8$ & $0.3-356.1$ & $40-78$ \\
\hline & & (179) & $(11.3)$ & $(260)$ & (27.9) & (18.1) & (64) \\
\hline 8 & Colorado & $105-150$ & $10.0-30.0$ & $10-1000$ & $11.4-26.0$ & $10-10000$ & $46.0-69.7$ \\
\hline 9 & $\mathrm{La} P a z$ & $134-216$ & $7.8-22.0$ & $1-950$ & $18.3-32.0$ & $0.1-98.0$ & $35-68$ \\
\hline 10 & Barco & $95-270$ & $2.0-23$ & $0.5-2000$ & $23.4-45.0$ & $0.02-33$ & $59-86$ \\
\hline
\end{tabular}

Valores en paréntesis corresponde a la media aritmética.

Fuente: (Ecopetrol S.A, 2010) 
En la Tabla 3 se presenta un resumen de las características de las formaciones más grandes del país en términos de OOIP, propiedades usadas como parámetros de selección para determinar la posible aplicación de nuevas tecnologías de recobro químico. Los datos muestran el alto grado de heterogeneidad de las formaciones, donde los rangos de las propiedades son amplios, por ejemplo el caso de la formación Carbonera con variaciones considerables en temperatura, permeabilidad y viscosidad del crudo; caso contrario a la formación Colorado, donde por ejemplo la temperatura presenta un rango de menor variación.

El análisis del potencial de aplicación de nuevas tecnologías de recobro en yacimientos colombianos continúa con el estudio de las ventanas de operación de las nuevas tecnologías de recobro en contraste con las propiedades de los yacimientos colombianos. Los principales parámetros de screening a considerar incluyen las propiedades del crudo como gravedad API y viscosidad; también propiedades del yacimiento como permeabilidad, profundidad, temperatura $\mathrm{y}$ saturación de fluidos. Adicionalmente, y dependiendo de la tecnología, será importante conocer la mineralogía del yacimiento y la composición del crudo. Para el caso de estudio presentado en este artículo, se presenta el análisis de las propiedades que se relacionan directamente con las potenciales mejoras de las nuevas tecnologías (temperatura, permeabilidad, viscosidad y gravedad API). (Delgadillo Aya et al, 2018).

\section{Rangos de aplicación de las nuevas tecnologías de recobro químico en los campos colombianos}

Para las nuevas tecnologías de recobro mejorado, a diferencia del screening de tecnologías convencionales, no se tiene un sustento en un gran número de proyectos ejecutados en campo. Adicionalmente, no se cuenta con unos rangos de screening validados por expertos; por lo tanto, su valoración se realiza con base en ventanas de operación que fueron establecidas a partir de los resultados reportados en proyectos piloto y estudios a nivel de laboratorio, los cuales pueden considerarse promisorios, aunque con un alto grado de incertidumbre.

Nuevos polímeros. A continuación, se describe el análisis de las condiciones operacionales para las nuevas tecnologías de polímeros y su potencial aplicación en campos colombianos.
En la tabla 4 se observan los parámetros de screening de HPAM en la primera fila y en las inferiores se presentan los rangos que son modificados con la aplicación de nuevas tecnologías (temperatura, salinidad y Saturación de aceite residual). Recientes estudios sugieren que estos parámetros de screening deben ser actualizados con base en experiencias de proyectos a escala comercial en formaciones de mayor permeabilidad e hidrocarburos de mayor viscosidad (Manrique, Ahmadi \& Samani, 2017). Sin embargo, las tecnologías de polímeros mejorados amplían los rangos de aplicación, lo cual redunda en un incremento de activos que pueden ser susceptibles de aplicación de estos métodos de recobro.

Con base en los datos presentados en las tablas 3 y 4 , se realizó una comparación de las ventanas operativas de las tecnologías de polímeros y las propiedades de las formaciones colombianas. En este caso, la comparación se realizó entre el HPAM (polímero sintético convencional y comercialmente probado), polímeros mejorados y biopolímeros. El análisis se realizó comparando de forma independiente tres variables que afectan fuertemente el desempeño de estos procesos de recobro: la temperatura, la permeabilidad y la viscosidad del crudo.

A continuación, en la figura 3, se presenta un conjunto de tres gráficos, cada uno de ellos muestra los valores mínimo y máximo de la propiedad en cada formación indicando en diferentes tonos los rangos de aplicación de las tecnologías basadas en inyección de polímeros, estos últimos establecidos con base en las ventanas operativas de la tabla 4.

Según los gráficos anteriores, teniendo en cuenta la temperatura y viscosidad de los yacimientos colombianos, el HPAM tiene una aplicación limitada en el país, lo que hace que las nuevas tecnologías de polímeros expandan ese rango de posibles aplicaciones. En este sentido, los biopolímeros han presentado mejores condiciones de filtrabilidad que los polímeros sintéticos en pruebas de laboratorio, esto podría tener una favorabilidad en las condiciones de inyectividad en yacimientos con bajas permeabilidades. En general, con las tecnologías de polímeros mejorados estructuralmente, polímeros funcionalizados con nanotecnología y biopolímeros se amplía la ventana operacional, cubriendo un mayor número de yacimientos con potencial de aplicación de estas tecnologías emergentes. 
Tabla 4. Ventanas de aplicación propuestas para los polímeros mejorados Vs HPAM.

\begin{tabular}{|c|c|c|c|c|c|c|c|c|}
\hline \multirow[t]{4}{*}{ HРAМ* } & $10-150$ & $>15$ & $>10-800$ & $<9000$ & $<176$ & 50000 & $>50$ & $\begin{array}{l}\text { Arenisca } \\
\text { preferible }\end{array}$ \\
\hline & $<5400$ & - & $1,8-5500$ & - & $<248$ & 180000 & $>30$ & - \\
\hline & $<5400$ & $>9$ & $>100$ & - & $<275$ & 180000 & $>30$ & $\begin{array}{r}\text { Areniscas y } \\
\text { carbonatos }\end{array}$ \\
\hline & $<5400$ & $>9$ & $1,8-5500$ & - & $<248$ & 180000 & $>50$ & - \\
\hline
\end{tabular}

*(Taber, Martin y Seright, 1997)

**(Delgadillo Aya et al, 2018)
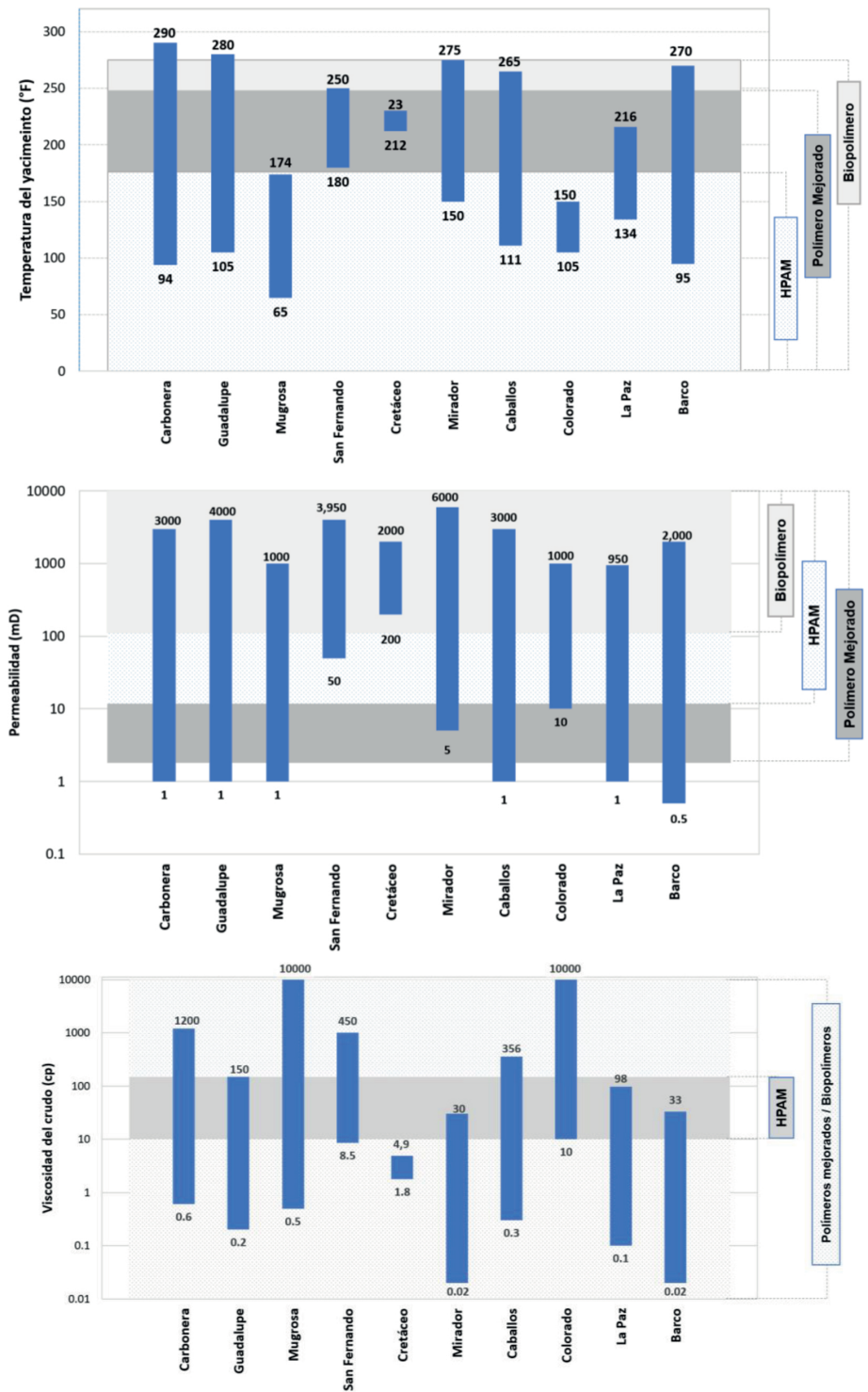

Figura 3. Ventanas de aplicación de tecnologías de polímeros en Colombia 
Nuevos Surfactantes. En el caso de los surfactantes, de acuerdo con la literatura, aunque los nuevos surfactantes no necesariamente alcanzan valores ultrabajos de tensión interfacial $\left(<10^{-3}\right.$ dinas $\left./ \mathrm{cm}\right)$, ofrecen mayor resistencia a la dureza y salinidad del agua de formación, por lo que representan una alternativa viable ante las dificultades actuales de los polímeros respecto a la degradación por efecto de corte $\mathrm{y} / \mathrm{o}$ altas salinidades (Zhu et al, 2013). En la tabla 5 se presentan los parámetros de screening de la inyección de surfactantes, así como la ventana operacional para los nuevos surfactantes, basada en pruebas realizadas a escala de laboratorio. En este caso se agruparon los criterios y se generó una sola tecnología llamada surfactantes mejorados en la que se incluyen los Smart surfactants, los surfactantes visco-elásticos y los surfactantes poliméricos; son tres tecnologías con ventanas de aplicación similares, con poca valoración experimental y amplio potencial, por lo cual se considera prometedora su evaluación.

Tabla 5. Ventanas de aplicación propuestas de los surfactantes Vs surfactantes mejorados

\begin{tabular}{|c|c|c|c|c|c|c|c|c|c|}
\hline $\begin{array}{c}\text { Inyección } \\
\text { surfactantes* }\end{array}$ & $<35$ & $>20$ & $>50$ & $>14.8$ & $<5300$ & $<155$ & NR & $>35$ & Arenisca \\
\hline
\end{tabular}

**(Taber, Martin y Seright, 1997)

**(Delgadillo Aya et al, 2018)

***(Chauhan, 2014)

De forma similar a la valoración realizada con las tecnologías de polímeros se realizó la comparación para las tecnologías de surfactantes. En la figura
4 se presentan las ventanas de aplicación para las tecnologías de surfactantes evaluando la temperatura y la gravedad API.

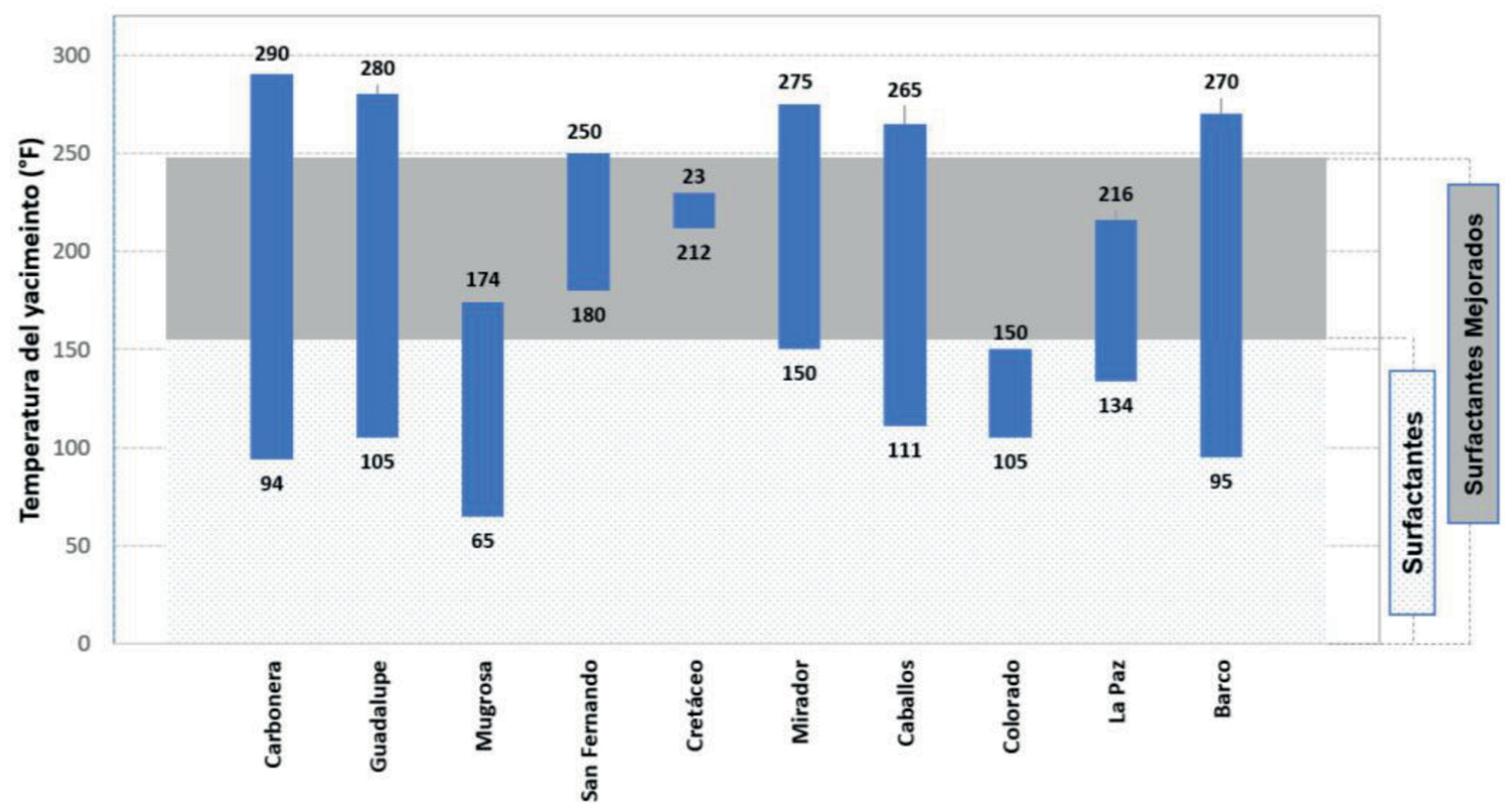




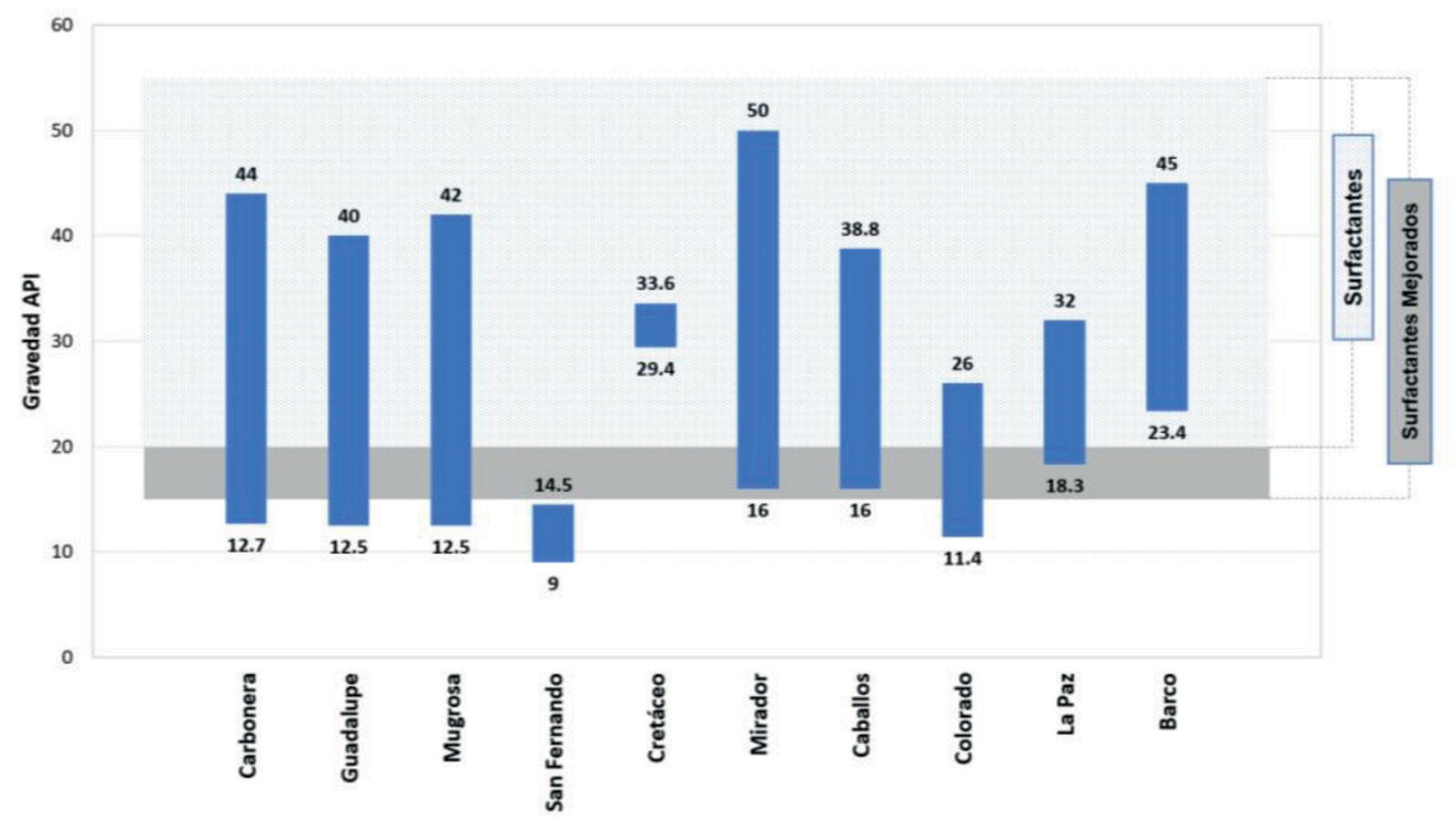

Figura 4. Ventanas de aplicación de tecnologías de surfactantes en Colombia

Con las tecnologías de surfactantes mejorados se impacta principalmente su eficiencia desde la disminución de tensiones ultra bajas a condiciones de alta temperatura hasta mejoras del número capilar, esto se debe a la combinación de reducciones menores de la tensión interfacial ( $\approx 1$ orden de magnitud) y aumentos de la viscosidad de los surfactantes poliméricos, restricción presentada con los surfactantes convencionales. Adicionalmente, es conocido que existen otras propiedades, como la salinidad del agua de formación, cuyo impacto es significativo en la degradación y la concentración miscelar crítica para los surfactantes; sin embargo, no se cuenta con información disponible de salinidad para todos los yacimientos colombianos que permita determinar la ventana operacional de estas tecnologías con relación a esta propiedad, por lo tanto, es un efecto que no fue posible evaluar en este trabajo.

\section{Crudo en sitio susceptible de aplicación de nuevas tecnologías de recobro químico}

Partiendo del análisis comparativo de las características de los yacimientos colombianos y las ventanas operativas propuestas para las nuevas tecnologías de recobro, se identifican de manera preliminar las formaciones en el país que cumplen con este novedoso screening técnico. Posteriormente, usando una base de datos que contiene la información de los campos colombianos (Ecopetrol S.A., EcoEOR, 2010), se calculó la cantidad de crudo en sitio correspondiente a los campos que cumplieron los criterios de screening para cada tecnología y que, por tanto, sería la cantidad de crudo impactado con la aplicación de las nuevas tecnologías de CEOR de aplicación. Los resultados de este análisis se muestran en la figura 5.

Comparado con la cantidad de crudo en sitio susceptible de aplicación para los procesos de inyección de polímero convencional, el cual se encuentra alrededor de 7000 millones de barriles, las tecnologías emergentes de recobro químico tienen un gran potencial de aplicación en los activos de Colombia, superando limitaciones técnicas, operacionales y de posibles sobrecostos por condiciones del crudo y de la calidad del agua de inyección. Lo anterior sugiere la posibilidad de contar con tecnologías que pueden contribuir con reposición de reservas e incrementen el factor de recobro de una manera costo-efectiva. Sin embargo, vale la pena resaltar, que la aplicación de una nueva tecnología de recobro químico por parte de cualquier empresa está condicionada a una viabilidad técnico-económica, que permita aprovechar las facilidades existentes en campo y evitar incurrir en altos costos (CAPEX) asociados al diseño e instalación de nuevas facilidades para el tratamiento, almacenamiento e inyección de los químicos seleccionados. 


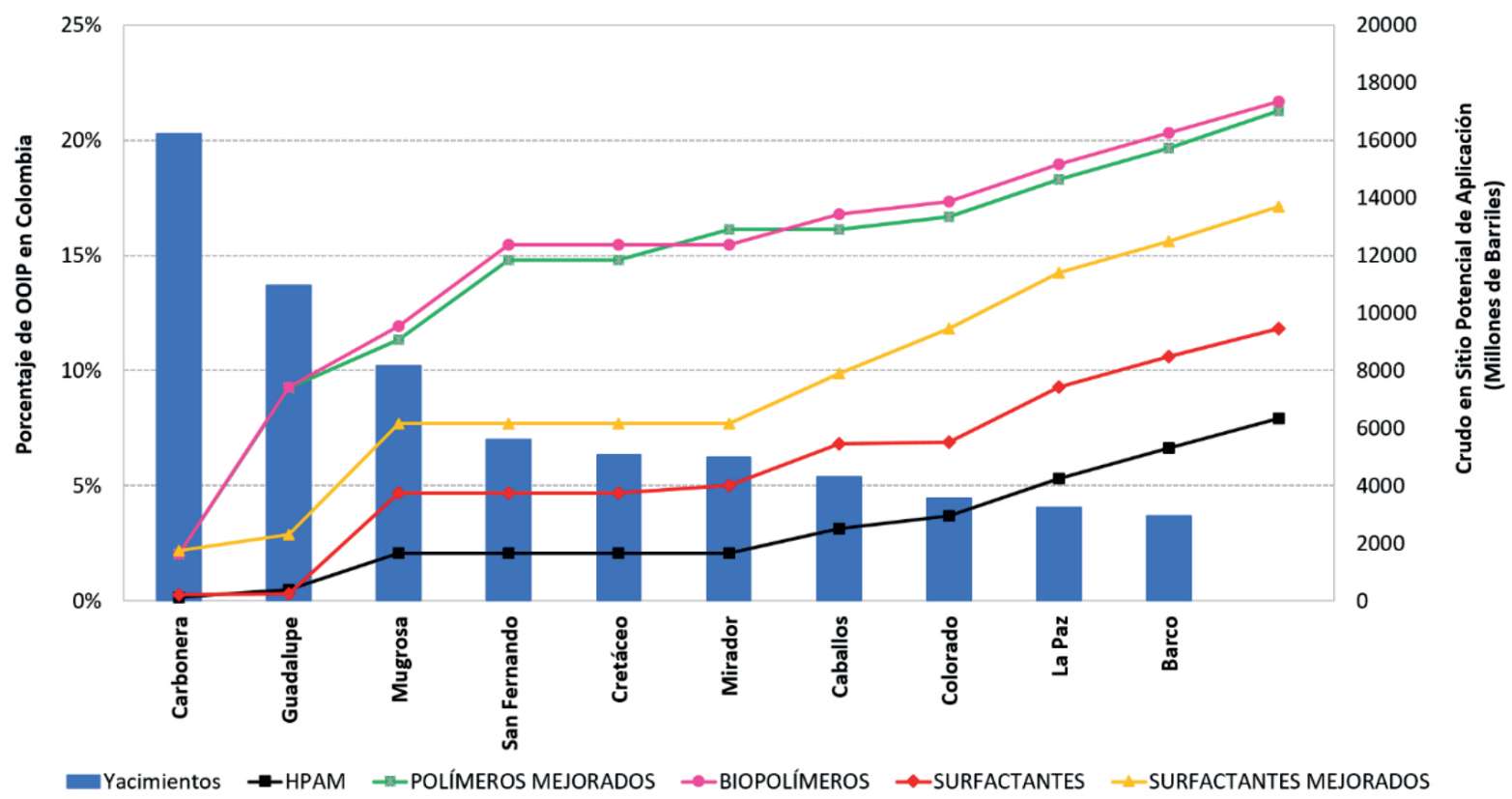

Figura 5. Crudo en sitio susceptible de aplicación de nuevas tecnologías emergentes de cEOR

\section{Conclusiones}

La inyección de polímeros HPAM tiene un rango de aplicación limitado debido a las condiciones de temperatura, salinidad y dureza de las aguas de formación, permeabilidad del intervalo productor y/o viscosidad. En Colombia se han implementado proyectos piloto de inyección de soluciones poliméricas tipo HPAM, las aplicaciones han sido desarrolladas para yacimientos con características específicas que justificaron su aplicabilidad en los campos evaluados. Sin embargo, existe un importante volumen de reservas que podrían ser objetivo de la aplicación de nuevas tecnologías de inyección de polímeros.

La inyección de surfactantes como mecanismo para atacar el aceite residual, solo reporta una aplicación en el país debido a los retos que implican la evaluación detallada de las interacciones fluidofluido y roca-fluidos es este tipo de procesos (ASP) y a la economía de los proyectos. Los avances investigativos de los últimos años han permitido el desarrollo de nuevas moléculas químicas capaces de superar las limitaciones técnicas de los surfactantes convencionales, relacionados principalmente con su mejor rendimiento en condiciones de alta temperatura y salinidad probadas a nivel de laboratorio.

Las tecnologías emergentes de recobro químico han permitido ampliar la ventana de operación de las tecnologías convencionales, aumentando el potencial de crudo en sitio susceptible para su aplicación, aspecto que puede contribuir a incrementar reservas y el incremento del factor de recobro en campos maduros sometidos a inyección de agua de una manera costo-efectiva.

Las nuevas tecnologías de recobro químico tienen un gran potencial de aplicación en los activos de Colombia superando las limitaciones técnicas, operacionales y de sobrecostos por condiciones del crudo y las características de los yacimientos. De los 7000 millones de barriles de crudo en sitio susceptible de aplicación de polímeros convencionales, el país pasaría a tener entre 14000 y 18000 millones de barriles de crudo en sitio susceptibles de aplicación de las nuevas tecnologías de recobro químico, condicionado a un estudio de viabilidad técnico-económica; por esto se recomienda continuar evaluando su comercialidad.

\section{Agradecimientos}

Agradecemos a Ecopetrol S.A., especialmente al Instituto Colombiano del Petróleo (ICP) quienes en convenio con la Universidad Industrial de Santander (UIS) por medio del acuerdo Marco No. 5222395 permitieron el desarrollo de esta investigación y la publicación de la misma. 


\section{Referencias bibliográficas}

Ahmadi, M. A., \& Shadizadeh, S. R. (2013). Implementation of a high-performance surfactant for enhanced oil recovery from carbonate reservoirs. Journal of Petroleum Science and Engineering, 110, 66-73.

Albonico, P., \& Lockhart, T. P. (1993, January). Divalent ion-resistant polymer gels for hightemperature applications: syneresis inhibiting additives. In SPE International Symposium on Oilfield Chemistry. Society of Petroleum Engineers.

Alvarado, V., Manrique, E. (2010). Enhanced Oil Recovery: Field Planning and Development Strategies. ISBN 978-1-85617-855-6, Elsevier, U.S.A

Amaya, M., Amaya, R., Castaño, H., Lozano, E., Rueda, C. F., Elphick, J., ... \& Marín, A. J. V. (2010). Casabe: Revitalización de un campo maduro. Oilfield review, 22(1).

ANH (2010). Información General de Cuencas Sedimentarias de Colombia

ANH (2018). Estadísticas de Producción Mensual de Petróleo. Colombia: Agencia Nacional de Hidrocarburos. Recuperado de http://www.anh. gov.co/Operaciones-Regalias-y-Participaciones/ Sistema-Integrado-de-Operaciones/Paginas/ Estadisticas-de-Produccion.aspx

Araujo, Y. C. \& Araujo, M. (2018). Polymers for application in high temperature and high salinity reservoirs - critical review of properties and aspects to consider for laboratory screening. Revista Fuentes: El reventón energético, 16(2), 55-71. DOI: http://dx.doi.org/10.18273/revfue. v16n2-2018004.

Barrero, D., Pardo, A., Vargas, C. A., \& Martínez, J. F. (2007). Colombian sedimentary basins: Nomenclature, boundaries and petroleum geology, a new proposal. Agencia Nacional de Hidrocarburos, 1, 92.

Bennetzen, M. V., \& Mogensen, K. (2014, December). Novel applications of nanoparticles for future enhanced oil recovery. In International petroleum technology conference. International Petroleum Technology Conference.

British Petroleum, B. P. (2017). Statistical Review of World Energy, 2010.

Castro, R., Maya, G., Mantilla, J., Diaz, V., Amaya, R., Lobo, A., ... \& Villar, A. (2014, May). Waterflooding in Colombia: Past, present, and future. In SPE Latin America and Caribbean Petroleum Engineering Conference. Society of Petroleum Engineers.

Castro-Garcia, R. H., Maya-Toro, G. A., Jimenez-Diaz, R., Quintero-Perez, H. I., Díaz-Guardia, V. M., Colmenares-Vargas, K. M., ... \& Pérez-Romero, R. A. (2016). Polymer flooding to improve volumetric sweep efficiency in waterflooding processes. CT\&F-Ciencia, Tecnología y Futuro, 6(3), 71-90.

Castro, R. H., Maya, G. A., Sandoval, J., Leon, J. M., Zapata, J. F., Lobo, A., ... \& Romero, J. L. (2013, July). Colloidal Dispersion Gels (CDG) in Dina Cretáceos Field: From Pilot Design to Field Implementation and Performance. In SPE Enhanced Oil Recovery Conference. Society of Petroleum Engineers.

Castro, R., Pérez, R., Maya, G., Quintero, H., Jimenez, R., García, H., \& Quintero, L. (2016). Polymer Flooding Process to Increase Recovery Factor. GEORESURSY, 18(4), 271-280.

Chauhan, P. D. (2014). Data analysis and summary for surfactant-polymer flooding based on oil field projects and laboratory data. Masters Theses. Missouri University of Science and Technology

Delgadillo Aya, C. L., Díaz Guardia, V. M., Maya Toro, G. A., Castro García R. H. y Quintero Pérez, H. I. (2018). Metodología para la priorización de tecnologías emergentes de recobro mejorado químico. Revista Fuentes: El reventón energético, 16(2), 41-53.

Donaldson, E. C., Chilingarian, G. V., \& Yen, T. F. (Eds.). (1989). Enhanced oil recovery, II: Processes and operations. Elsevier.

Dupuis, G., Rousseau, D., Tabary, R., \& Grassl, B. (2011). Flow of hydrophobically modified water- 
soluble-polymer solutions in porous media: New experimental insights in the diluted regime. SPE Journal, 16(01), 43-54.

Ecopetrol S.A. (2010). EcoEOR. Software para la Selección de Procesos EOR

El-Hoshoudy, A. N., Desouky, S. E. M., Elkady, M. Y., Al-Sabagh, A. M., Betiha, M. A., \& Mahmoud, S. (2017). Hydrophobically associated polymers for wettability alteration and enhanced oil recoveryArticle review. Egyptian Journal of Petroleum, 26(3), 757-762.

Hernandez, F. A. T., Niño, J. C. L., \& Moreno, R. L. (2018). Effects of salts and temperature on rheological and viscoelastic behavior of low molecular weight HPAM solutions. Fuentes, el reventón energético, 16(1), 19-35.

Jensen, T., Kadhum, M., Kozlowicz, B., Sumner, E. S., Malsam, J., Muhammed, F., \& Ravikiran, R. (2018, April). Chemical EOR Under Harsh Conditions: Scleroglucan As A Viable Commercial Solution. In SPE Improved Oil Recovery Conference. Society of Petroleum Engineers.

Kamal, M. S., Sultan, A. S., Al-Mubaiyedh, U. A., \& Hussein, I. A. (2015). Review on polymer flooding: rheology, adsorption, stability, and field applications of various polymer systems. Polymer Reviews, 55(3), 491-530.

Kamal, M. S., Hussein, I. A., \& Sultan, A. S. (2017). Review on surfactant flooding: phase behavior, retention, IFT, and field applications. Energy \& Fuels, 31(8), 7701-7720.

Lake, L. W. (1996). Enhanced Oil Recovery. Prentice Hall

Leonhardt, B., Ernst, B., Reimann, S., Steigerwald, A., \& Lehr, F. (2014, April). Field testing the polysaccharide schizophyllan: results of the first year. In SPE Improved Oil Recovery Symposium. Society of Petroleum Engineers.

Lizcano-Nino, J. C., de Sousa Ferreira, V. H., \& Moreno, R. B. L. (2020). Less-concentrated HPAM solutions as a polymer retention reduction method in CEOR. Fuentes, el reventón energético, 18(1), 75-92.
Maya-Toro, G. A., Castro-García, R. H., PachónContreras, Z. D. P., \& Zapata-Arango, J. F. (2012). Polymer gels for controlling water thief zones in injection wells. CT\&F-Ciencia, Tecnología y Futuro, 5(1), 37-44.

Maya, G., Jimenez, R., Castro, R., Mantilla, J., Vargas, J., Cardenas, F., ... \& Romero, J. (2015, November). Design and implementation of the first polymer flooding project in Colombia: Yariguí-Cantagallo Field. In SPE Latin American and Caribbean Petroleum Engineering Conference. Society of Petroleum Engineers.

Maya, G., Castro, R., Sandoval, J., Pachon, Z., Jimenez, R., Pinto, K., ... \& Muñoz, S. (2014, May). Successful polymer gels application in a highly channeled peripheral injection well: Tello Field pilot. In SPE Latin America and Caribbean Petroleum Engineering Conference. Society of Petroleum Engineers.

Maya, G. A., Mercado Sierra, D. P., Castro, R., Trujillo Portillo, M. L., Soto, C. P., \& Pérez, H. (2010, January). Enhanced Oil Recovery (EOR) StatusColombia. In SPE Latin American and Caribbean Petroleum Engineering Conference. Society of Petroleum Engineers.

Molano, A. M. J., Navarro, S. F. M., \& Díaz, R. J. (2014). Metodología para el diseño de baches en un proceso de inyección de polímeros para recobro mejorado, considerando fenómenos de interacción roca/ fluidos. Fuentes: El reventón energético, 12(2), 6.

Manrique, E., Ahmadi, M. \& Samani, S. (2017). Historical and Recent Observations in Polymer Floods: An Update Review. CT\&F - Ciencia Tecnología y Futuro 6(5), 17-48. https://doi. org/10.29047/issn.0122-5383.

Olajire, A. A. (2014). Review of ASP EOR (alkaline surfactant polymer enhanced oil recovery) technology in the petroleum industry: Prospects and challenges. Energy, 77, 963-982.

Perez, R., Castro Garcia, R. H., Jimenez, R., Maya, G. A., Leon Hinestrosa, J. M., Reyes, J. D., ... \& Quintero Roa, L. M. (2017, May). Mature Field Revitalization Using Polymer Flooding: Palogrande-Cebú Field Case. In SPE Latin America and Caribbean Petroleum Engineering Conference. Society of Petroleum Engineers. 
Pye, D. J. (1964). Improved secondary recovery by control of water mobility. Journal of Petroleum technology, 16(08), 911-916.

Pinto, M. S., Herrera, D. M., \& Angarita, J. C. G. (2018). Production optimization for a conceptual model through combined use of polymer flooding and intelligent well technology under uncertainties. Fuentes, el reventón energético, 16(1), 37-45.

Quadri, S. M. R., Jiran, L., Shoaib, M., Hashmet, M. R., AlSumaiti, A. M., \& Alhassan, S. M. (2015, November). Application of biopolymer to improve oil recovery in high temperature high salinity carbonate reservoirs. In Abu Dhabi international petroleum exhibition and conference. Society of Petroleum Engineers.

Raffa, P., Broekhuis, A. A., \& Picchioni, F. (2016). Polymeric surfactants for enhanced oil recovery: A review. Journal of Petroleum Science and Engineering, 145, 723-733.

Raffa, P., Wever, D. A. Z., Picchioni, F., \& Broekhuis, A. A. (2015). Polymeric surfactants: synthesis, properties, and links to applications. Chemical reviews, 115(16), 8504-8563.

Reichenbach-Klinke, R., Stavland, A., Strand, D., Langlotz, B., \& Brodt, G. (2016, March). Can associative polymers reduce the residual oil saturation?. In SPE EOR Conference at Oil and Gas West Asia. Society of Petroleum Engineers.

Rosenkilde, C., Brakstad, K., \& Smith, J. B. (2016, May). Degradation of Synthetic Polymers during Radial Injection in a Sandstone. In 78th EAGE Conference and Exhibition 2016.

Sandiford, B. B. (1964). Laboratory and field studies of water floods using polymer solutions to increase oil recoveries. Journal of Petroleum Technology, 16(08), 917-922.

Seright, R. S. (2016, April). How much polymer should be injected during a polymer flood?. In SPE Improved Oil Recovery Conference. Society of Petroleum Engineers.

Shaefer, M. T., Carman, P. S., \& Ngoma, V. (2011, January). New thermo thickening associative polymer system pushes limits of surfactant gels. In SPE Hydraulic Fracturing Technology
Conference. Society of Petroleum Engineers

ShamsiJazeyi, H., Miller, C. A., Wong, M. S., Tour, J. M., \& Verduzco, R. (2014). Polymer-coated nanoparticles for enhanced oil recovery. Journal of Applied Polymer Science, 131(15).

ShamsiJazeyi, H., Verduzco, R., \& Hirasaki, G. J. (2014). Reducing adsorption of anionic surfactant for enhanced oil recovery: Part I. Competitive adsorption mechanism. Colloids and Surfaces A: Physicochemical and Engineering Aspects, 453, 162-167.

Sheng, J. (2010). Modern chemical enhanced oil recovery: theory and practice. Gulf Professional Publishing.

Sheng, J. J., Leonhardt, B., \& Azri, N. (2015). Status of polymer-flooding technology. Journal of Canadian Petroleum Technology, 54(02), 116126.

Song, B., Hu, X., Shui, X., Cui, Z., \& Wang, Z. (2016). A new type of renewable surfactants for enhanced oil recovery: dialkylpolyoxyethylene ether methyl carboxyl betaines. Colloids and Surfaces A: Physicochemical and Engineering Aspects, 489, 433-440.

Sorbie, K. S. (1991). Polymer-Improved Oil Recovery, 115 Glasgow. Scotland: Blackie \& Son, 126-163.

Spildo, K., Johannessen, A. M., \& Skauge, A. (2012, January). Low salinity waterflood at reduced capillarity. In SPE Improved Oil Recovery Symposium. Society of Petroleum Engineers.

Standnes, D. C., \& Skjevrak, I. (2014). Literature review of implemented polymer field projects. Journal of Petroleum Science and Engineering, 122, 761-775.

Sveistrup, M., van Mastrigt, F., Norrman, J., Picchioni, F., \& Paso, K. (2016). Viability of biopolymers for enhanced oil recovery. Journal of Dispersion Science and Technology, 37(8), 1160-1169.

Sun, G., Crouse, B., Freed, D. M., Xu, R., Bautista, J., Zhang, R., \& Dressler, M. (2018). Polymer flooding-Does Microscopic Displacement Efficiency Matter. Fuentes, el reventón energético, 16(2). 
Taylor, K. C., \& Nasr-El-Din, H. A. (1998). Watersoluble hydrophobically associating polymers for improved oil recovery: A literature review. Journal of Petroleum Science and Engineering, 19(3-4), 265-280.

Thomas, S. (2008). Enhanced oil recovery-an overview. Oil \& Gas Science and TechnologyRevue de l'IFP, 63(1), 9-19.

Tadros, T. (2007). Colloid stability using polymeric surfactants. Colloid Stability, 235-262.

Vanegas, P. A. V., Ruiz, T. Y. Z., Macualo, F. H. E., \& Martin, C. A. G. (2019). Metodología para la formulación de proyectos de recuperación química mediante analogías. Revista Fuentes, 17(1), 29-35.

Viñarta, S. C., Delgado, O. D., Figueroa, L. I., \& Fariña, J. I. (2013). Effects of thermal, alkaline and ultrasonic treatments on scleroglucan stability and flow behavior. Carbohydrate polymers, 94(1), 496-504.

Wu, Y., Mahmoudkhani, A., Watson, P., Fenderson, T. R., \& Nair, M. (2012, January). Development of new polymers with better performance under conditions of high temperature and high salinity. In SPE EOR conference at oil and gas West Asia. Society of Petroleum Engineers.

Wu, X. C., Xiong, C., Han, D., Liu, H., Gao, S., Xu, H., ... \& Ren, F. (2014, October). A New IOR Method for Mature Waterflooding Reservoirs:" Sweep Control Technology". In SPE Asia Pacific Oil \& Gas Conference and Exhibition. Society of Petroleum Engineers.
Wu, X., Xiong, C., Xu, H., Zhang, J., Lu, C., Lu, X., ... \& Chen, J. (2015, November). A novel particletype polymer and IOR/EOR property evaluation. In Abu Dhabi International Petroleum Exhibition and Conference. Society of Petroleum Engineers.

Wu, X., Yang, Z., Xu, H., Zhang, L., Xiong, C., Yang, H., ... \& Cao, H. (2016, April). Success and Lessons Learned from Polymerflooding a Ultra High Temperature and Ultra High Salinity Oil Reservoir-A Case Study from West China. In SPE Improved Oil Recovery Conference. Society of Petroleum Engineers.

Yang, F., Wang, D., Yang, X., Sui, X., Chen, Q., \& Zhang, L. (2004, January). High concentration polymer flooding is successful. In SPE Asia Pacific Oil and Gas Conference and Exhibition. Society of Petroleum Engineers.

Zaitoun, A., \& Potie, B. (1983, January). Limiting conditions for the use of hydrolyzed polyacrylamides in brines containing divalent ions. In SPE Oilfield and Geothermal Chemistry Symposium. Society of Petroleum Engineers.

Zhu, Y., Hou, Q., Liu, W., Ma, D., \& Liao, G. Z. (2012, January). Recent progress and effects analysis of ASP flooding field tests. In SPE Improved Oil Recovery Symposium. Society of Petroleum Engineers.

Fecha de recepción: 25 de Noviembre de 2019

Fecha de aceptación: 21 de Octubre de 2020 